\section{Bacteria fight it out}

Interspecies competition at mucosal surfaces can select for the evolution of invasive traits, according to a recent report in Current Biology.

At mucosal surfaces, pathogenic and commensal members of the microbiota must compete with each other. In a mouse model of infection, Haemophilus influenzae can outcompete Streptococcus pneumoniae in the nasopharynx by stimulating neutrophil-mediated complementopsonized killing (opsonophagocytosis). S. pneumoniae has more than 90 capsular serotypes, which differ in protein structure and have varying responses to complement deposition. In virulent isolates of $S$. pneumoniae, which can cause invasive disease, the capsule confers resistance to opsonophagocytosis.

Elena Lysenko and colleagues were interested in whether S. pneumoniae virulence, or capsule type, is affected by the competition with $H$. influenzae. Mathematical modelling of the within-host dynamics of $H$. influenzae co-infection with different strains of $S$. pneumoniae predicted that $H$. influenzae can completely outcompete an opsonophagocytosissusceptible $S$. pneumoniae $\left(\mathrm{P}_{\mathrm{S}}\right)$ strain and that the presence of $H$. influenzae drives selection for an invasive S. pneumoniae $\left(\mathrm{P}_{\mathrm{R}}\right)$ strain encoding a capsule that confers resistance to opsonophagocytosis. In a direct competition experiment comparing two pneumococcal isolates, a $\mathrm{P}_{\mathrm{R}}$ isolate (serotype T4) was competitively inferior to a $\mathrm{P}_{\mathrm{S}}$ isolate (serotype $\mathrm{T} 23 \mathrm{~F}$ ), indicating that expression of the resistant capsule has a fitness cost. However, in the presence of $H$. influenzae, expression of the more resistant capsule is advantageous - the density of the $\mathrm{P}_{\mathrm{S}}$ strain declined steeply within 24 hours of inoculation, whereas there was no decline in the density of the $\mathrm{P}_{\mathrm{R}}$ strain. These effects could be reversed by switching the capsule type. Finally, a three-way competition experiment between $H$. influenzae and the two S. pneumoniae strains showed that the presence of a sufficient burden of
H. influenzae enhances colonization by the $\mathrm{P}_{\mathrm{R}}$ strain and inhibits colonization by the $\mathrm{P}_{S}$ strain, as predicted by the theoretical model.

As pneumococcal transmission occurs during the asymptomatic phase of infection, the factors driving the evolution of costly capsule serotypes that function during the symptomatic phase of infection have been unclear. These results indicate that within-host competition between $H$. influenzae and S. pneumoniae could be a contributing factor.

\section{Sheilagh Molloy}

ORIGINAL RESEARCH PAPER Lysenko, E. S. et al. Within-host competition drives selection for the capsule virulence determinant of Streptococcus pneumoniae. Curr. Biol. 17 Jun 2010 (doi: 10.1016/ j.cub.2010.05.051)

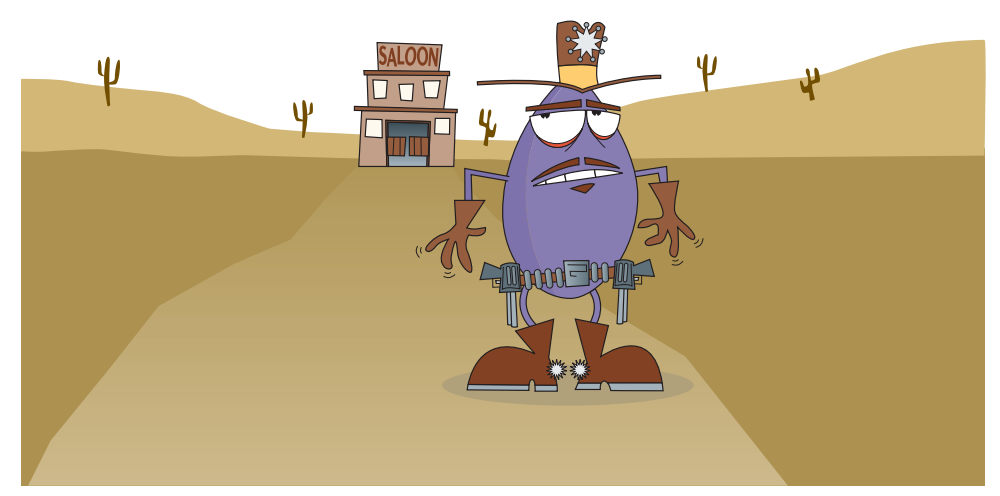

